\title{
Aplicação da Técnica de Fio Quente na Determinação das Propriedades Térmicas de Polímeros Fundidos
}

\author{
Wilson N. dos Santos \\ Departamento de Engenharia de Materiais, UFSCar
}

\begin{abstract}
Resumo: A natureza da estrutura molecular dos plásticos torna as propriedades desses materiais fortemente dependentes da temperatura. A condutividade térmica, difusividade térmica e o calor específico, conhecidos como propriedades térmicas, são as três propriedades físicas mais importantes de um material em cálculos de transferência de calor. Valores confiáveis dessas propriedades são essenciais em polímeros, tanto em problemas envolvendo estado estacionário como em estado não estacionário de troca de calor. Hoje, várias técnicas diferentes para a determinação da condutividade térmica e difusividade térmica podem ser encontradas na literatura. Neste trabalho, a técnica de fio quente paralelo é empregada na determinação experimental das propriedades térmicas de polímeros. Três polímeros semi-cristalinos (PEAD, PEBD e PP), e dois polímeros amorfos (PS e HIPS) foram selecionados para este trabalho. As medidas experimentais são feitas desde a temperatura ambiente até a, aproximadamente, $50^{\circ} \mathrm{C}$ acima da temperatura de fusão. As amostras são preparadas pelo processo de extrusão, partindo-se do pó ou "pellets" do polímero no estado sólido. Um molde especial de aço inoxidável, em forma de paralelepípedo retangular, provido de isoladores cerâmicos entre o fio quente, o termopar e o próprio molde é utilizado para armazenar a massa polimérica fundida, cujas propriedades térmicas serão medidas. Os resultados experimentais obtidos são comparados com dados disponíveis na literatura e com dados fornecidos pelos fabricantes. Uma análise crítica desta metodologia mostra as vantagens e desvantagens desta técnica experimental em relação à técnica de pulso de energia.
\end{abstract}

Palavras-chave: Polímero fundido, condutividade térmica, difusividade térmica, calor específico, propriedades térmicas, técnica de fio quente.

\section{Hot Wire Technique in the Determination of Thermal Properties of Melt Polymers}

\begin{abstract}
The nature of the molecular structure of the plastics makes the properties of such materials markedly temperature dependent. Thermal conductivity, thermal diffusivity and specific heat, namely the thermal properties, are the three most important physical properties of a material that are needed for heat transfer calculations. Reliable thermal properties values are essential for polymers, both in steady and non-steady state situations. Nowadays, several different techniques for the determination of the thermal diffusivity and thermal conductivity may be found in the literature. In this work, the hot wire parallel technique is employed in the experimental determination of the thermal properties of polymers. Three semi-crystalline polymers (HDPE, LDPE and PP), and two amorphous polymers (PS and HIPS) were selected for this work. Samples are prepared through the extrusion process starting from the powder or pellets of the solid polymer. A special mould of stainless steel in the shape of a rectangular parallelepiped provided with ceramic insulators between the hot wire, thermocouple and the mould is employed to store the melt extruded polymer whose thermal properties will be measured. Measurements are carried out from room temperature up to approximately $50{ }^{\circ} \mathrm{C}$ above the melting point. Experimental results obtained are checked against data found in literature and those ones furnished by the manufacturers. A critical analysis of this method shows the advantages and disadvantages of this technique when compared with the laser flash technique.
\end{abstract}

Keywords: Melt polymer, thermal conductivity, thermal diffusivity, specific heat, thermal properties, hot wire technique.

\section{Introdução}

No decorrer dos últimos anos, a modelagem matemática tornou-se uma ferramenta essencial na melhoria do controle do processo e da qualidade do produto, no processamento de polímeros. À medida em que os modelos matemáticos se tornam mais sofisticados, há um aumento na necessidade de dados confiáveis das propriedades térmicas dos polímeros. Essas propriedades são observadas quando calor é adicionado ou removido do material e se tornam importantes em qualquer projeto que precise funcionar em qualquer ambiente térmico. Um exemplo bastante simples e corriqueiro é

Autor para correspondência: Wilson N. dos Santos, Departamento de Engenharia de Materiais, UFSCar, Via Washington Luiz km 235 - Caixa Postal 676, CEP: 13565-905, São Carlos, SP. E-mail: dwns@power.ufscar.br 
a tampa plástica de uma garrafa térmica, que precisa ser isolante (baixa condutividade térmica) para armazenar o seu conteúdo quente ou frio. Ela não pode armazenar calor (baixo calor específico) e não pode sofrer grandes variações em suas dimensões (baixo coeficiente de expansão térmica) quando resfriada ou aquecida, o que causaria perda de calor ou dificuldade para abri-la. Por outro lado, a cobertura plástica sobre a superfície sinuosa de um motor elétrico precisa ter alta condutividade térmica para permitir a transferência de calor, mas não pode expandir quando aquecida se há pequenos espaços disponíveis. Desde que mudanças na estrutura polimérica (mudanças no peso molecular, cristalinidade, transição vítrea ou ramificações da cadeia e adição de carga) causam mudanças nas propriedades térmicas, a medida dessas propriedades pode, também, ser usada para ajudar a entender a relação estrutura polimérica/propriedade ${ }^{[1]}$.

A condutividade térmica de polímeros é bem baixa, quando comparada com a condutividade de materiais metálicos, e de alguns materiais cerâmicos. Do ponto de vista de processamento, a baixa condutividade térmica cria alguns problemas reais: ela limita a taxa com a qual o polímero pode ser aquecido e plastificado ${ }^{[2]}$. No resfriamento, a baixa condutividade pode provocar não uniformidade e encolhimento. Isso pode resultar em tensões de congelamento, deformação do extrudado, delaminação, vazios de moldado, etc. A condutividade térmica de um material plástico sólido depende da cristalinidade do material e, portanto, da história térmica prévia. Essa propriedade é, também, afetada pela anisotropia da amostra e, quando existe anisotropia, é recomendado que se façam medidas adicionais apropriadas ${ }^{[3]}$.

O calor específico de polímeros amorfos aumenta linearmente com a temperatura abaixo e acima da temperatura de transição vítrea (Tg), sendo que ao redor dessa temperatura ocorre uma variação brusca dessa propriedade. No caso de polímeros semicristalinos essa variação em Tg é menos pronunciada, ocorrendo, entretanto, um máximo distinto no ponto de fusão cristalino. Para uma estrutura cristalina perfeitamente uniforme o calor específico é teoricamente infinito no ponto de fusão. Desde que esse não é o caso de polímeros semicristalinos, esses materiais exibem no ponto de fusão um pico com uma certa largura. Quanto mais estreito for o pico mais uniforme é a morfologia cristalina. Acima do ponto de fusão o calor específico aumenta suavemente com a temperatura.

A difusividade térmica é importante em problemas envolvendo estado não-estacionário, o que ocorre durante o aquecimento e resfriamento de um polímero. Em processamento é uma propriedade fundamental, no processo de moldagem por injeção, para a determinação do tempo de ciclo de moldagem. Na análise da maioria dos problemas de extrusão ela é considerada constante, embora na realidade ela dependa da pressão, temperatura e orientação.

Essas três propriedades estão relacionadas entre si pela equação:

$$
\alpha=\frac{\mathrm{k}}{\rho \mathrm{c}_{\mathrm{p}}}
$$

onde: $\alpha=$ difusividade térmica $\left(\mathrm{m}^{2} / \mathrm{s}\right), \mathrm{k}=$ condutividade tér- mica $(\mathrm{W} / \mathrm{mK}), \rho=$ densidade volumétrica ou massa específica $\left(\mathrm{kg} / \mathrm{m}^{3}\right), \mathrm{c}_{\mathrm{p}}=$ calor específico a pressão constante( $\left.\mathrm{J} / \mathrm{kg} \mathrm{K}\right)$.

Hoje em dia, várias técnicas diferentes para a determinação da condutividade térmica e difusividade térmica podem ser encontradas na literatura ${ }^{[4]}$. Neste trabalho, utilizando-se a técnica de fio quente, são medidas experimentalmente essas propriedades para três polímeros semi-cristalinos (PEAD, PEBD e PP), e dois polímeros amorfos (PS e HIPS).

\section{0 método de fio quente}

O método do fio quente, descrito por Schieirmacher ${ }^{[5]} \mathrm{em}$ 1888 é um método absoluto, direto e não estacionário. Sua primeira aplicação prática foi reportada em 1949 por Van der Held e Van Drunen ${ }^{[6]}$, na determinação da condutividade térmica de líquidos. Todavia foi Haupin ${ }^{[7]}$ que em 1960 utilizou pela primeira vez essa técnica na determinação da condutividade térmica de materiais cerâmicos. No Brasil essa técnica foi introduzida por Santos ${ }^{[8]}$ em 1986 na determinação das propriedades térmicas de cerâmicas refratárias.

Para uma formulação matemática do método, o fio quente é assumido ser uma fonte de calor ideal (massa $=0$ e, portanto, capacidade térmica $=0$ ), infinitamente longa e fina (diâmetro $=$ 0 ), a qual é circundada até o infinito pelo material cuja condutividade térmica pretende-se determinar ${ }^{[9]}$. Esta hipótese implica que o transiente de temperatura que é registrado pelo termopar, no ponto de medida, durante o experimento, não pode ser alterado pelo fato da amostra real ter dimensões finitas. Ao passar uma corrente elétrica constante através do fio, uma quantidade constante de calor, por unidade de tempo e por unidade de comprimento, é liberada pelo fio e vai se propagar através do material. Essa propagação de calor num meio infinito gera, no material, um campo transiente de temperaturas.

$\mathrm{Na}$ prática, a fonte teórica linear é aproximada por uma resistência elétrica fina e o sólido infinito é substituído por uma amostra finita. Assim sendo, a capacidade térmica do fio, a resistência de contato entre ele e a amostra e o tamanho finito da amostra são fatores que impõem um tempo mínimo e um tempo máximo a serem utilizados no cálculo da condutividade térmica. Os fundamentos teóricos, bem como detalhes desta técnica experimental, já foram descritos em várias ocasiões ${ }^{[10,11]}$.

A técnica de fio quente paralelo, uma das quatro possíveis variações do método de fio quente, é empregada neste trabalho. Nesta técnica, aplicando-se uma corrente elétrica constante através do fio, e registrando-se o transiente de temperatura no ponto de medida Mp localizado a uma distância $\mathrm{r}$ do fio, a condutividade térmica e o calor específico são calculados de acordo com a seguinte equação:

$$
\mathrm{k}=\frac{-\mathrm{q}}{4 \pi \mathrm{T}(\mathrm{t})} \mathrm{E}_{\mathrm{i}}\left(\frac{-\rho \mathrm{c}_{\mathrm{p}} \mathrm{r}^{2}}{4 \mathrm{kt}}\right)
$$

onde: $\mathrm{k}=$ condutividade térmica $(\mathrm{W} / \mathrm{mK}), \mathrm{q}$ ' $=$ densidade linear de potência $(\mathrm{W} / \mathrm{m}), \mathrm{T}=$ excesso de temperatura em relação à temperatura inicial de referência $(\mathrm{K}), \rho=$ densidade volumétrica ou massa específica do material $\left(\mathrm{kg} / \mathrm{m}^{3}\right), \mathrm{c}_{\mathrm{p}}=$ calor específico à 
pressão constante $(\mathrm{J} / \mathrm{kgK}), \mathrm{r}=$ distância radial a partir do fio quente $(\mathrm{m}), \mathrm{t}=$ tempo, contado a partir do início da liberação de calor (s) e-Ei = função exponencial integral. A distância r recomendada entre o fio quente e o termopar é de 15 a $17 \mathrm{~mm}$.

A condutividade térmica e o calor específico são determinados a partir de um duplo ajuste por regressão não linear ${ }^{[11]}$, obtendo-se assim, essas duas propriedades simultaneamente, a partir do mesmo transiente térmico experimental. Conhecendo-se então a densidade do material, a difusividade térmica pode então ser calculada pela equação 1 . O sistema de aquisição e processamento de dados utilizado neste trabalho é totalmente

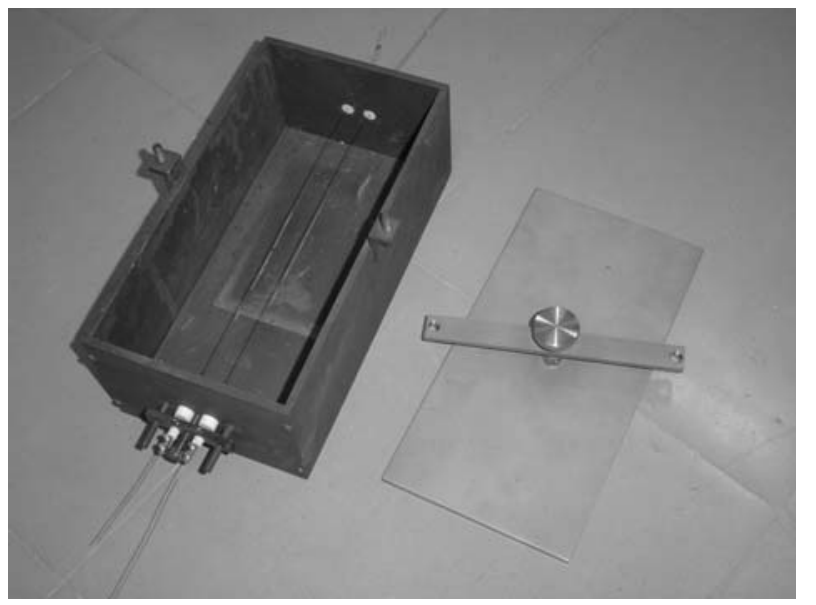

Figura 1. Molde de aço inoxidável.

Tabela 1. Conjuntos de amostras.

\begin{tabular}{|c|c|c|c|c|c|}
\hline Material & Aplicação [12,13] & $\begin{array}{l}\text { Temperatura de } \\
\text { transição vítrea } \\
\qquad\left({ }^{\circ} \mathrm{C}\right)^{[13]}\end{array}$ & $\begin{array}{l}\text { Temperatura de fusão } \\
\text { cristalina/amolecimento } \\
\left({ }^{\circ} \mathbf{C}\right)^{[13]}\end{array}$ & $\begin{array}{c}\text { Densidade } \\
\text { do sólido } \\
\text { a } 25^{\circ} \mathrm{C}\left(\mathrm{kg} / \mathrm{m}^{3}\right)\end{array}$ & $\begin{array}{c}\text { Cristalinidade } \\
(\%)^{[13]}\end{array}$ \\
\hline PEAD & $\begin{array}{l}\text { Contentores, material hospitalar, } \\
\text { bombonas, fita-lacre de } \\
\text { embalagens. }\end{array}$ & -120 & 135 & 955 & até 95 \\
\hline PEBD & $\begin{array}{l}\text { Filmes e frascos para } \\
\text { embalagens de produtos } \\
\text { alimentícios, farmacêuticos e } \\
\text { químicos, utensílios domésticos, } \\
\text { brinquedos. }\end{array}$ & -20 & 120 & 915 & até 60 \\
\hline PP & $\begin{array}{l}\text { Pára-choque de automóveis, } \\
\text { carcaças de eletrodomésticos, } \\
\text { recipientes em geral, brinquedos, } \\
\text { tubos para canetas } \\
\text { esferográficas, material } \\
\text { hospitalar, carpetes, sacarias. }\end{array}$ & $4-12$ & $165-175$ & 901 & $60-70$ \\
\hline PS & $\begin{array}{l}\text { Utensílios domésticos rígidos, } \\
\text { brinquedos, escovas, embalagens } \\
\text { para cosméticos e alimentos, } \\
\text { placas expandidas. }\end{array}$ & 100 & 160 & 1048 & amorfo \\
\hline HIPS & $\begin{array}{l}\text { Artigos de uso geral: copos, } \\
\text { bandejas, embalagens } \\
\text { descartáveis, artigos } \\
\text { eletrodomésticos e de escritório, } \\
\text { indústria automobilística. }\end{array}$ & 100 & 160 & 1048 & amorfo \\
\hline
\end{tabular}

automatizado: o transiente de temperatura que é detectado pelo termopar é processado em um microcomputador, via um conversor analógico-digital, usando-se para isso um programa computacional especialmente desenvolvido para esse objetivo.

\section{Preparação das amostras}

Cinco conjuntos de amostras, três polímeros semi-cristalinos (PEAD, PEBD e PP), e dois polímeros amorfos (PS e HIPS) foram preparados através do processo de extrusão a partir do pó ou "pellet" do polímero sólido. Um molde especial de aço inoxidável com dimensões internas de (220x100x65) $\mathrm{mm}$ em forma de paralelepípedo retangular, provido de isoladores cerâmicos entre o fio quente, o termopar e o próprio molde é utilizado para armazenar o polímero extrudado fundido, cujas propriedades térmicas serão medidas. Após o preenchimento do molde, o mesmo é recoberto com papel alumínio e levado ao forno a temperatura máxima de medida desejada, cerca de aproximadamente $50{ }^{\circ} \mathrm{C}$ acima da temperatura de fusão, e deixado a essa temperatura por algumas horas até que toda a massa polimérica entre em equilíbrio térmico nessa temperatura. Uma vez atingido esse equilíbrio o processo de medida experimental das propriedades térmicas é então iniciado, em temperaturas decrescente até a temperatura ambiente, com o intervalo de temperaturas desejado. A finalidade da cobertura do molde com papel alumínio é minimizar a degradação do material na superfície externa superior, quando não se tem atmosfera controlada no interior do forno. A Figura 1 mostra o molde utilizado e a Tabela 1 mostra algumas características das amostras ensaiadas. 


\section{Resultados e Discussão}

Utilizando-se o procedimento descrito anteriormente, foi então medida experimentalmente a condutividade térmica e o calor específico dos polímeros selecionados, desde a temperatura máxima selecionada até a temperatura ambiente. As Figuras 2 e 3 mostram os resultados experimentais obtidos para a condutividade térmica e o calor específico para o conjunto de polímeros estudados. As Figuras 4 a 7 mostram os resultados experimentais obtidos para a condutividade térmica e o calor específico para cada grupo de polímeros separadamente, bem como os resultados encontrados na literatura e também alguns resultados fornecidos pelos próprios fabricantes. As correspondentes difusividades térmicas podem então ser calculadas a partir das condutividades térmicas e calores específicos medidos experimentalmente e das densidades dos polímeros, usando-se para isso a equação 1 .

A Figura 2 mostra a condutividade térmica em função da temperatura para os cinco polímeros estudados, três semicristalinos e dois amorfos. Para cada um dos dois grupos há uma tendência comum no comportamento dessas curvas em função da temperatura. Para o grupo dos semi-cristalinos (PEAD, PEBD e PP) a condutividade térmica decresce com o aumento da temperatura, desde a temperatura ambiente até o ponto de fusão do polímero, parecendo haver uma tendência de aumento da condutividade térmica com o aumento da temperatura a partir do ponto de fusão. Essa figura mostra também que a forma da curva condutividade térmica versus temperatura é a mesma, estando deslocada para cima ou para baixo no sentido de condutividades térmicas maiores ou menores na razão direta das densidades dos polímeros. Esse comportamento fica mais evidenciado observando-se a Figura 4. Para o grupo dos polímeros amorfos (PS e HIPS), cujas densidades são iguais, a condutividade térmica se mantém aproximadamente constante com o aumento da temperatura até o ponto de amolecimento e, a partir daí, aumenta suavemente com o aumento da temperatura. Esses detalhes são mostrados na Figura 5.

A Figura 3 mostra o calor específico em função da temperatura para os mesmos polímeros. Aqui também duas ten-

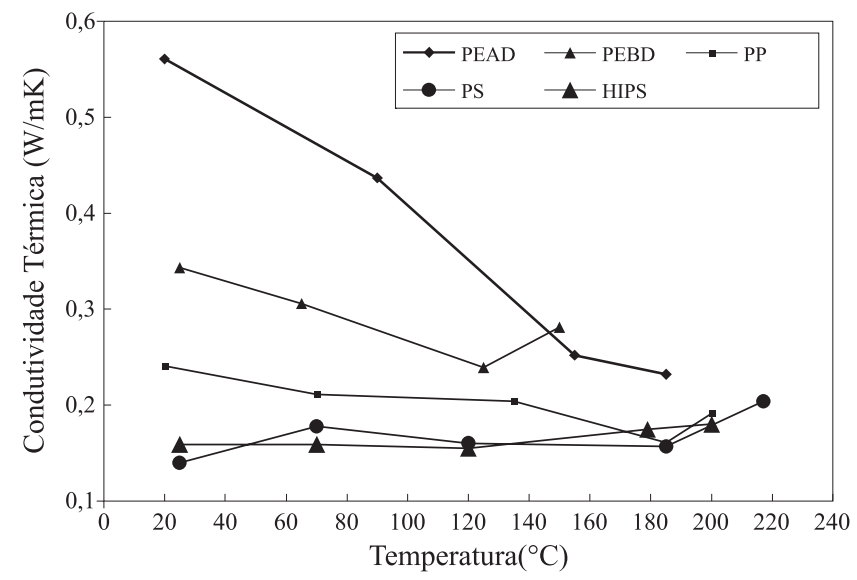

Figura 2. Condutividade térmica em função da temperatura.

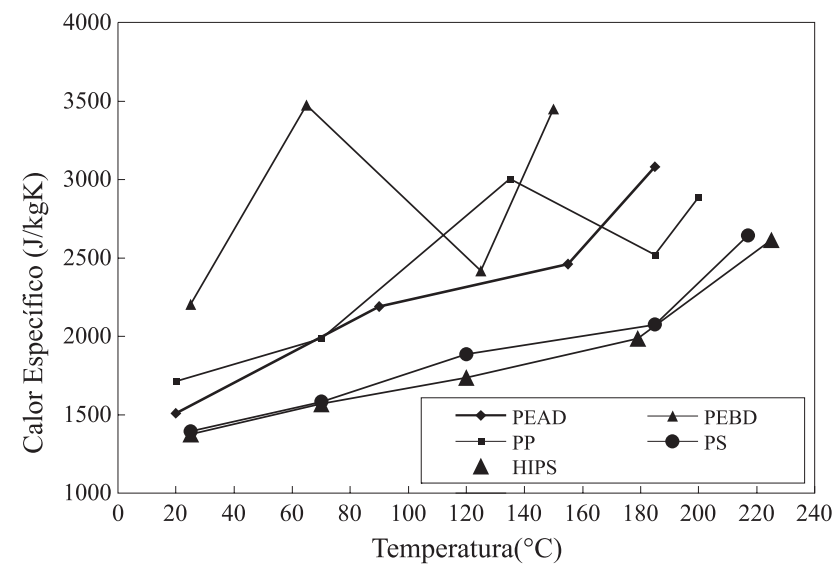

Figura 3. Calor específico em função da temperatura.

dências diferentes podem ser observadas. Para os polímeros amorfos o calor específico aumenta com o aumento da temperatura em toda a faixa de temperaturas estudadas, sendo que acima do ponto de amolecimento do polímero esse aumento do calor específico com a temperatura é mais acentuado. Detalhes são melhores observados na Figura 6. Já para o grupo dos polímeros semi-cristalinos, o comportamento do calor específico em função da temperatura é diferente. Ele aumenta com o aumento da temperatura até o ponto de fusão do polímero. Após atingir o estado fundido o calor específico diminui bruscamente, passando a aumentar novamente com o aumento da temperatura, conforme mostra a Figura 7.

De acordo com Rauwendaal ${ }^{[2]}$, além de haver diferentes técnicas de medidas das propriedades de polímeros, diferenças consideráveis nessas mesmas propriedades podem ocorrer para um particular polímero devido a variações na distribuição de peso molecular, aditivos, história termomecânica, etc. No caso das propriedades térmicas, dados conflitantes muitas vezes são encontrados para alguns polímeros. Para outros há uma escassez completa de dados, enquanto que ainda para outros polímeros muitas vezes são encontrados dados dessas propriedades, sem haver, entretanto, qualquer referência quanto à temperatura na qual essa propriedade foi medida.

Para o poliestireno foi possível fazer uma comparação entre os resultados obtidos por esta técnica experimental, dados encontrados na literatura e também dados fornecidos

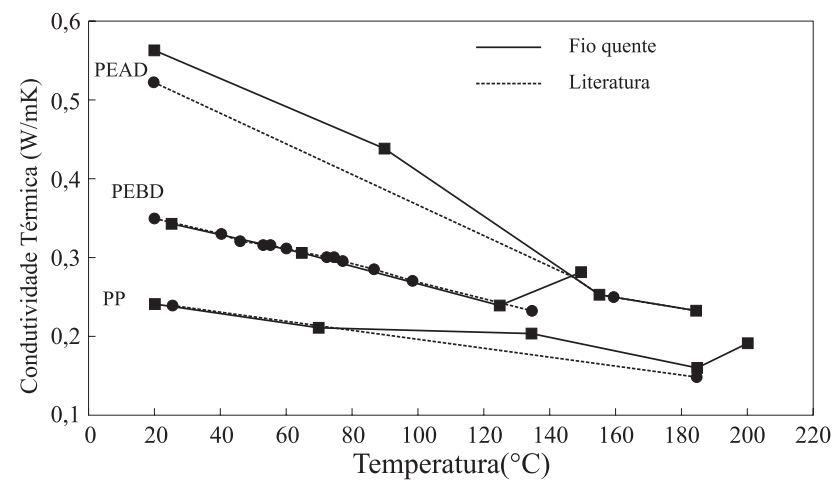

Figura 4 Condutividade térmica: polímeros semi-cristalinos. 


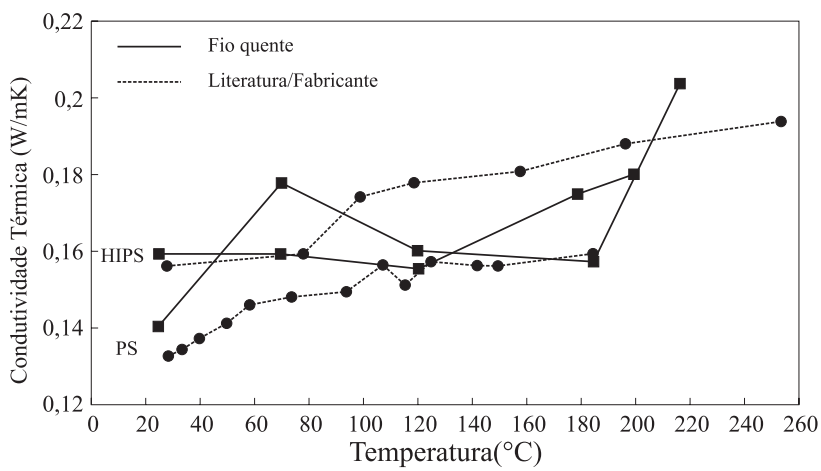

Figura 5. Condutividade térmica: polímeros amorfos.
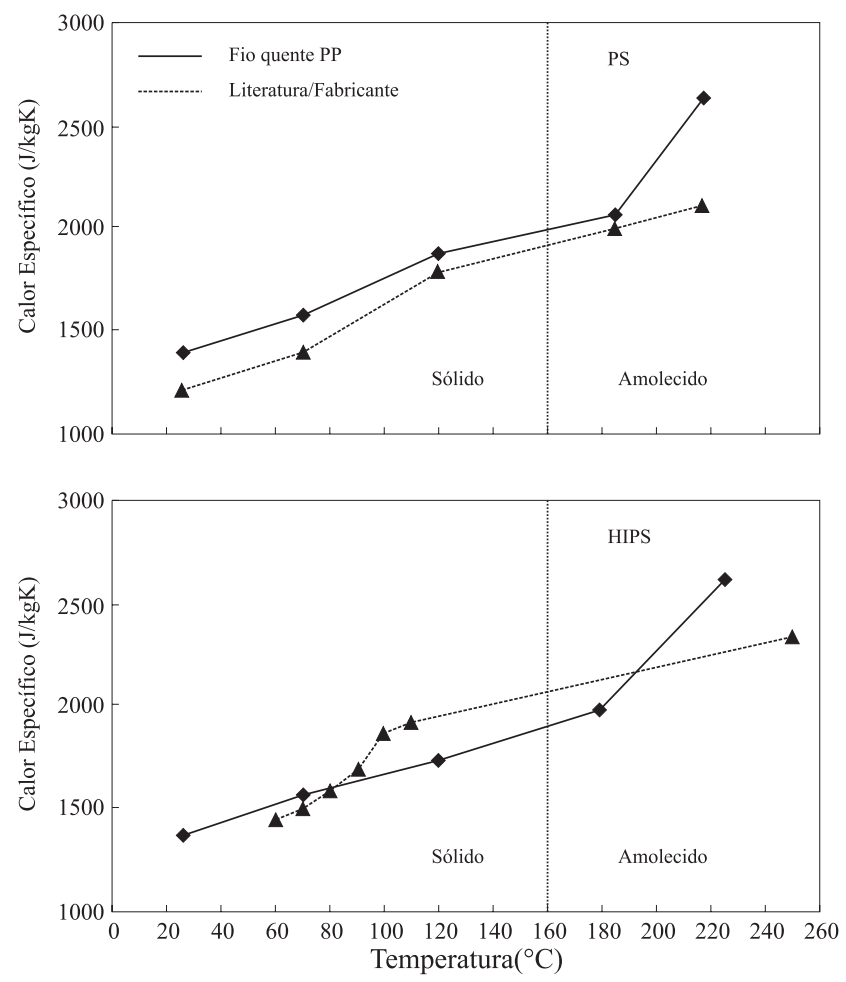

Figura 6. Calor específico: polímeros amorfos.

pelo próprio fabricante, num amplo intervalo de temperaturas: desde a temperatura ambiente até $220^{\circ} \mathrm{C}$. As Figuras 5 e 6 mostram que os resultados obtidos pela técnica de fio quente estão em excelente concordância com os dados encontrados na literatura e aqueles fornecidos pelo fabricante. Para o HIPS essa comparação foi possível apenas com dados fornecidos pelo fabricante. Para esse material a concordância entre os resultados experimentais obtidos e aqueles fornecidos pelo fabricante também é muito boa, conforme também mostram as Figuras 5 e 6.

Para o PEBD também foi possível fazer uma comparação entre os resultados de condutividade térmica obtidos pela técnica de fio quente e dados encontrados na literatura. A figura 4 mostra também uma excelente concordância entre os resultados obtidos por esta técnica e dados encontrados na literatura.

Já para os outros dois polímeros estudados (PEAD, e PP), essa comparação só foi possível em algumas temperaturas es-
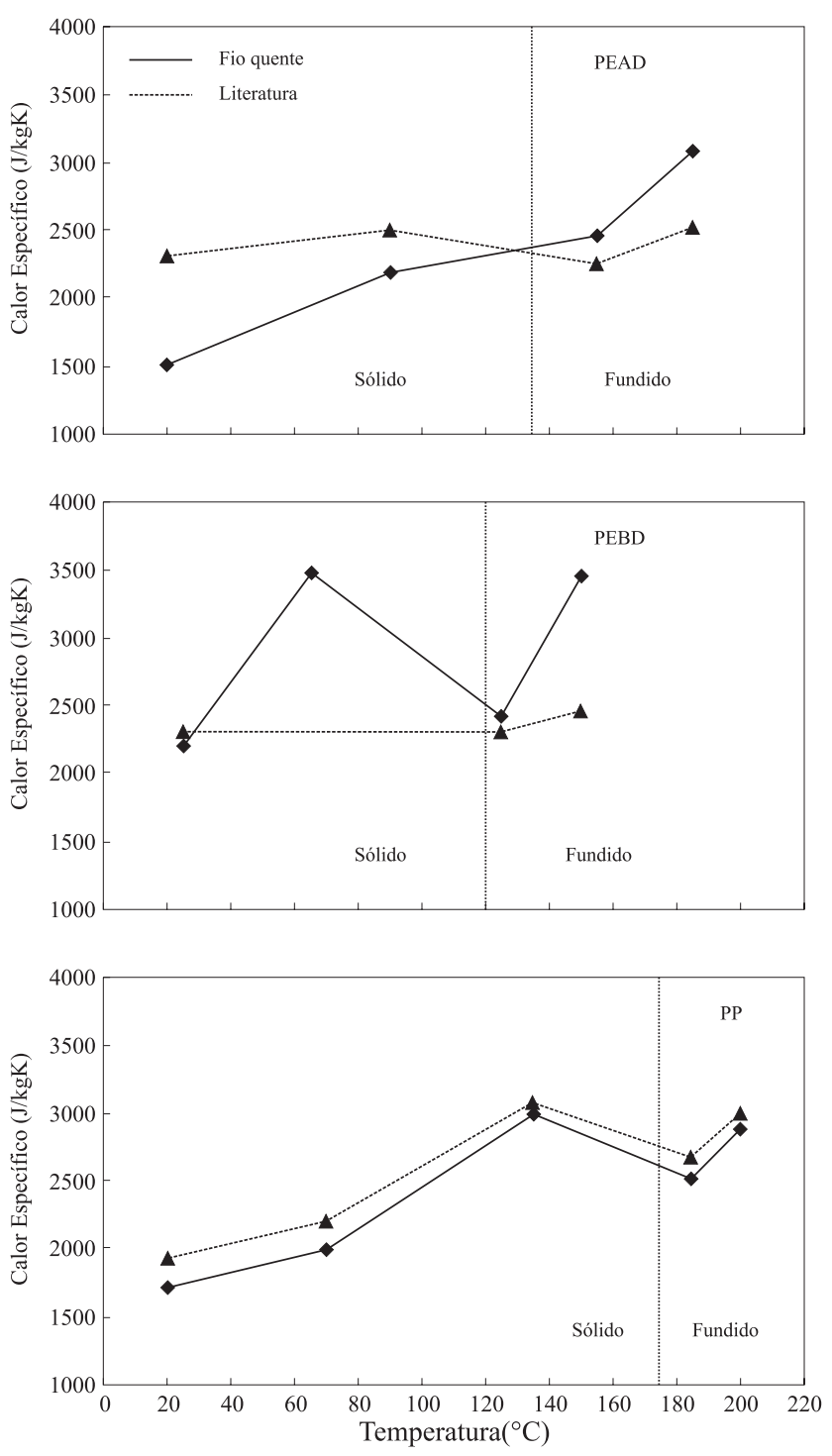

Figura 7. Calor específico: polímeros semi-cristalinos.

pecíficas. Para esses polímeros pode-se dizer que, para a condutividade térmica, a concordância entre os resultados experimentais obtidos por esta técnica e os dados encontrados na literatura é excelente, conforme mostra a Figura 4, enquanto que para o calor específico há algumas discrepâncias em determinadas temperaturas, fato este que é ilustrado na Figura 7.

Face aos resultados experimentais obtidos, pode-se afirmar que o método do fio quente mostrou-se bastante adequado para a determinação das propriedades térmicas de polímeros, tanto no estado sólido, quanto no estado fundido. $\mathrm{O}$ que poderia ser colocado como uma desvantagem desta técnica em relação à técnica de pulso de energia, por exemplo, é o tamanho das amostras. Como o tamanho mínimo da amostra, tamanho esse que propicie um intervalo de tempo suficiente para a aquisição de dados confiáveis, é diretamente proporcional à difusividade térmica ${ }^{[14]}$, isto significa na prática que as dimensões mínimas de amostras para o caso de polímeros podem ser drasticamente reduzidas em relação às dimensões utilizadas neste trabalho, que são as dimensões 
usuais para materiais cerâmicos. Conhecendo-se aproximadamente o intervalo de variação da difusividade térmica dos polímeros é possível determinar as dimensões mínimas dos corpos de prova para os materiais poliméricos nesta técnica experimental. Outra desvantagem desta técnica em relação à técnica de pulso de energia, e também associada ao tamanho da amostra, é o tempo necessário para a mesma atingir o equilíbrio a uma dada temperatura. No processo de medida das propriedades térmicas em função da temperatura nesta técnica experimental, o intervalo de tempo entre a medida a uma temperatura e outra chega a ser de 10 horas. Isto acontece devido a baixa difusividade térmica dos polímeros e a grande massa de material envolvida. Ao se diminuir o tamanho das amostras, conseqüentemente esse intervalo de tempo também diminuirá. Com isto é possível aumentar o universo de polímeros aos quais essa técnica possa ser aplicada, e diminuir também o tempo total de ensaio em qualquer intervalo de temperaturas desejado. Entretanto, o método do fio quente tem duas grandes vantagens em relação à técnica de pulso de energia. A primeira delas é que nesta técnica, a partir do mesmo experimento são determinadas simultaneamente a partir do mesmo transiente de temperaturas a condutividade térmica, a difusividade térmica e o calor específico, enquanto que na técnica de pulso de energia é determinada apenas a difusividade térmica. A segunda grande vantagem é o custo de implementação desta técnica, que pode chegar a apenas cerca de $30 \%$ do custo da técnica de pulso de energia, além da sua implementação ser bastante rápida.

Sempre que uma nova técnica é proposta, a avaliação do desvio experimental envolvido é fundamental. Nesta técnica, as incertezas podem ser classificadas segundo dois tipos: aquelas introduzidas pelo procedimento de medida e registro do transiente de temperatura, e as incertezas devido ao procedimento de análise por regressão não linear. No caso da primeira fonte, o desvio no resultado final obtido para uma determinada propriedade $\mathrm{P}$ pode ser dado por ${ }^{[15]}$ :

$\delta \mathrm{P}=\left[\left(\frac{\partial \mathrm{P}}{\partial \mathrm{x}_{1}} \delta \mathrm{x}_{1}\right)^{2}+\left(\frac{\partial \mathrm{P}}{\partial \mathrm{x}_{2}} \delta \mathrm{x}_{2}\right)^{2}+\left(\frac{\partial \mathrm{P}}{\partial \mathrm{x}_{3}} \delta \mathrm{x}_{3}\right)^{2}+\cdots+\left(\frac{\partial \mathrm{P}}{\partial \mathrm{x}_{\mathrm{n}}} \delta \mathrm{x}_{\mathrm{n}}\right)^{2}\right]^{1 / 2}$

onde $\mathrm{P}=\mathrm{P}\left(\mathrm{x}_{1}, \mathrm{x}_{2}, \mathrm{x}_{3}, \ldots, \mathrm{x}_{\mathrm{n}}\right)$ é um resultado experimental calculado, que é função das variáveis independentes $\mathrm{x}_{1}, \mathrm{x}_{2}$, $\mathrm{x}_{3}, \ldots, \mathrm{x}_{\mathrm{n}}, \mathrm{P}$ é o desvio associado à propriedade $\mathrm{P}, \mathrm{e} \mathrm{x}_{1}, \mathrm{x}_{2}$, ' $\mathrm{x}_{3}, \ldots, \mathrm{x}_{\mathrm{n}}$ são as incertezas nas variáveis independentes. Utilizando-se então a equação 3 verifica-se que o desvio percentual introduzido pelo procedimento de registro e medida do transiente de temperatura é da ordem de $\pm 5 \%$. O programa computacional que faz a análise de regressão não linear com ajuste por mínimos quadrados não calcula diretamente o desvio no parâmetro ajustado, associado a esse ajuste. Entretanto, para se fazer uma estimativa da qualidade do ajuste é calculado o coeficiente de correlação R em cada uma das medidas experimentais, o qual é dado por ${ }^{[15]}$ :

$$
R=\frac{\sum_{i=1}^{N} y_{i}(\text { exp }) y_{i}(\text { esp })-\frac{1}{N} \sum_{i=1}^{N} y_{i}(\text { exp }) \sum_{i=1}^{N} y_{i}(\text { esp })}{\left\{\left[\sum_{i=1}^{N}\left(y_{i}(\exp )\right)^{2}-\frac{1}{N}\left(\sum_{i=1}^{N} y_{i}(\exp )\right)^{2}\right] \times\left[\sum_{i=1}^{N}\left(y_{i}(\text { esp })\right)^{2}-\frac{1}{N}\left(\sum_{i=1}^{N} y_{i}(\text { esp })\right)^{2}\right]\right\}^{1 / 2}}
$$

onde $\mathrm{y}_{\mathrm{i}}(\exp )$ são valores experimentais, $\mathrm{y}_{\mathrm{i}}(\mathrm{esp})$ são valores esperados partir do ajuste e $\mathrm{N}$ é o número total de pontos experimentais.

Neste trabalho, os diversos coeficientes de correlação calculados variaram entre $\mathrm{R}=0,993$ e $\mathrm{R}=0,999$. Sabendo-se que a correlação perfeita corresponde a $\mathrm{R}=1$, conclui-se que $\mathrm{o}$ desvio associado ao procedimento de análise por regressão não linear é muito pequeno, quando comparado com os desvios introduzidos pelo procedimento de medida e registro do transiente de temperatura. Assim sendo, o desvio total no cálculo das propriedades térmicas utilizando o equipamento e o procedimento proposto neste trabalho é estimado como sendo da ordem de $\pm 5 \%$.

A reprodutibilidade dos resultados, como já foi mostrado em outras ocasiões ${ }^{[11]}$ é muito boa. Entretanto, a densidade do polímero na temperatura em que o ensaio é feito, e que é um dado de entrada para o programa de ajuste, deve ser fornecido com a maior exatidão possível, pois o calor específico obtido no programa de ajuste é bastante sensível às variações desse parâmetro. Isso é perfeitamente explicável, uma vez que os materiais poliméricos podem sofrer grandes variações de volume (altos coeficientes de expansão térmica) com a variação da temperatura, variando significativamente a densidade do polímero, ou seja a massa do material por unidade de volume. Para exemplificar o que acaba de ser citado, seja considerado novamente o caso do poliestireno, já tomado anteriormente como exemplo. Sua densidade a temperatura ambiente é $1048 \mathrm{~kg} / \mathrm{m}^{3}$, e sua densidade a $217^{\circ} \mathrm{C}$ é $961 \mathrm{~kg} / \mathrm{m}^{3}$. Portanto, o parâmetro de entrada correto para o programa de ajuste nos cálculos a $217^{\circ} \mathrm{C}$ é $961 \mathrm{~kg} / \mathrm{m}^{3}$. Os resultados obtidos do ajuste são $0,2042 \mathrm{~W} / \mathrm{mK}$ para a condutividade térmica, $2641 \mathrm{~J} / \mathrm{kgK}$ para o calor específico, e $8,045 \times 10^{-8} \mathrm{~m}^{2} / \mathrm{s}$ para a difusividade térmica. Se for fornecido ao programa o valor da densidade do material à temperatura ambiente $\left(1048 \mathrm{~kg} / \mathrm{m}^{3}\right.$, e não $\left.961 \mathrm{~kg} / \mathrm{m}^{3}\right)$, não se fazendo, portanto, a correção da densidade em função da temperatura, os valores obtidos pelo programa de ajuste seriam $0,2032 \mathrm{~W} / \mathrm{mK}$ para a condutividade térmica, $2415 \mathrm{~J} / \mathrm{kgK}$ para o calor específico, e $8,013 \times 10^{-8} \mathrm{~m}^{2} / \mathrm{s}$ para a difusividade térmica, ou seja uma variação de $0,5 \%$ para a condutividade térmica, $9,3 \%$ para o calor específico, e $0,4 \%$ para a difusividade térmica. Isso mostra que a obtenção de valores confiáveis de calor específico está condicionada ao fornecimento preciso da densidade do polímero na temperatura de ensaio. Observa-se por outro lado que o valor da condutividade térmica calculado pelo programa de ajuste é pouco influenciado por alterações na densidade do material, o que significa na prática que, mesmo quando não se conhece o valor exato da densidade do material na temperatura de ensaio, pode-se obter valores confiáveis para a condutividade térmica.

\section{Agradecimentos}

O autor agradece à FAPESP (Proc. 2001/08225-4) e ao CNPq (Proc. 302969/2002-0) pelo apoio financeiro recebido. Agradece também ao Prof. Dr. José Augusto Marcondes Agnelli e ao Prof. Dr. José Alexandrino de Souza pelas discussões técnicas. 


\section{Referências Bibliográficas}

1. Encyclopedia of Polymer Science and Technology, Interscience Publishers, Vol. 13, John Wiley \& Sons Inc., New York (1970).

2. Rauwendaal, C.-“Polymer Extrusion”, Hanser Publishers, Carl Hanser Verlag, Munich (1986).

3. Ogorkiewicz, R.M.-"Thermoplastics-Properties and Design”, John Wiley \& Sons, London (1974).

4. Santos, W.N. dos, - Cerâmica, 48, 306, p.86 (2002).

5. Schieirmacher, A.L., - Wiedemann Ann Phys, 34, p.38 (1888).

6. Van Der Held, E.F.M. \& Van Drunen, F.G., - Physics, 15, 10, p.865 (1949).

7. Haupin, W.E., Am Ceram Soc Bull, 39, 3, p.139 (1960).

8. Santos, W.N. dos \& Cintra Filho, J.S., - Cerâmica, 32, 198, p.151 (1986).
9. Carslaw, H.S., Jaeger, J.C.-“Conduction of Heat in Solids”, Oxford University Press, Oxford (1959).

10. Santos, W.N. dos \& Cintra Filho, J.S., - Cerâmica, 37, 252, p.101 (1991).

11. Santos, W.N. dos \& Gregório Filho, R., - Polímeros: Ciência e Tecnologia, 14, 5, p. 354 (2004).

12. Biasoto, E.M.-“Polímeros como Materiais de Engenharia", Edgard Blucher Ltda., São Paulo (1991).

13. Biasoto, E.M. \& Mendes, L.C.-“Introdução a Polímeros”, Edgard Blucher Ltda., São Paulo (1999).

14. Santos, W.N. dos \& Gregório Jr., R., - Cerâmica, 49, 309, p.29 (2003).

15. Holman, J.P.-“ Experimental Methods for Engineers”, McGraw-Holl, New York (1971).

Enviado: 17/01/05

Reenviado: 05/05/05

Aprovado: 23/05/05 